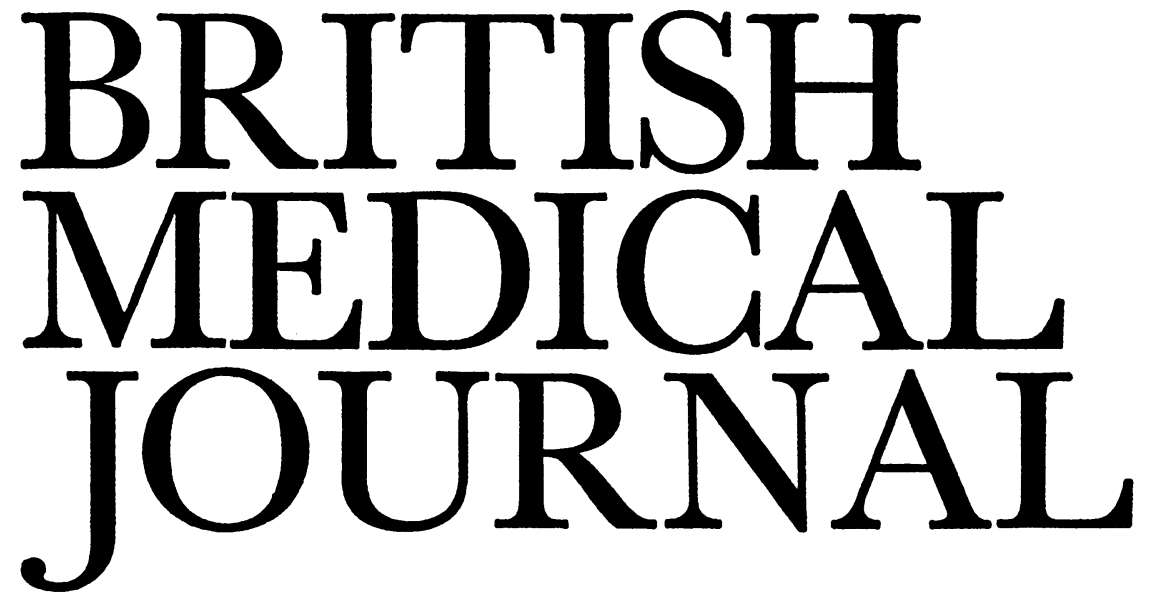

LONDON SATURDAY 19 JANUARY 1974

\title{
Ultrasounding the Heart
}

Despite a slow start after its introduction in 1954 by I. Edler and C. H. Hertz ${ }^{1}$ echocardiography has developed rapidly over the last five years. It is still of increasing importance in cardiac diagnosis.

Echocardiography causes no discomfort and needs little co-operation from the patient, so it is suitable even on young children and infants. No ionizing irradiation is used, and the pulsed ultrasound beam exposes the patient to no hazard. It has steadily gained in precision both through technical advances and through having been checked against data obtained from catheterization, angiocardiography, and surgery. The echocardiogram (U.C.G.) is now highly reliable in the recognition and description of many forms of valve disease ${ }^{2-5}$ and is rapidly gaining ground in the diagnosis of congenital heart disease. ${ }^{6-8}$ Though weaker in quantitative assessment, echocardiography is beginning to challenge established invasive methods for measuring the size of the heart chambers and myocardial performance. ${ }^{910}$ It will undoubtedly overtake them as technical improvements increase the accuracy of recording and as the advantages of an undisturbed physiological state and the ability to repeat the examination of the patient without risk are recognized.

On 26 October the British Cardiac Society held a symposium on echocardiography at which doctors unfamiliar with the subject were introduced to the principles and techniques, the normal rnitral echogram, the changes in rheumatic mitral valve disease and in disorders not affecting the mitral valve, the investigation of pericardial disease, and the study of the movement and dimensions of the left ventricle.

Describing ultrasound techniques Dr. C. F. P. Wharton explained the physical principles and the apparatus generally used. Ultrasound pulses at a rate of $1,000 / \mathrm{sec}$ are aimed at the cardiac structure under investigation and reflected back to be demonstrated on an oscilloscope or as a continuous recording on paper.

Dr. Clive Layton described the appearance of the normal mitral echogram. ${ }^{11}$ The valve ring and each cusp may be studied, though the anterior mitral cusp is usually the main target, as it is most easily and reliably recorded. In diastole the anterior cusp of the valve opens rapidly and moves anteriorly into the left ventricle. This diastolic opening movement is an index of the velocity of blood entering the left ventricle.
Immediately after rapid opening the cusp begins to move slowly back towards the closed position, the diastolic closure movement, and reaches an intermediate position at the end of the phase of rapid ventricular filling. This intermediate position is retained until the onset of atrial systole, which flings the valve open to its maximum position once more. The subsequent return to the intermediate position during atrial diastole is usually interrupted by rapid complete closure with the onset of mechanical contraction of the left ventricle.

Measurement of the diastolic closure rate helps in assessing disorders of the mitral valve and in those affecting the left ventricular muscle. ${ }^{12}$ The diastolic closure rate of the anterior mitral cusp is affected by the rate of left ventricular filling. This rate is slow when the mitral valve is obstructed or when diastolic distensibility is reduced by hypertrophy, as in severe aortic stenosis. Left ventricular filling is abnormally rapid when the stroke volume is increased, as in mitral regurgitation, or the left atrial pressure is high, as in constrictive pericarditis.

Dr. Christine Rodger described the appearance of the mitral echogram in rheumatic mitral disease. Thickening and scarring of the valve usually results in a diminished amplitude of excursion, while obstruction to the left ventricular filling causes the diastolic closure rate to be reduced below $50 \mathrm{~mm} /$ sec. In severe mitral stenosis the rate may be less than $15 \mathrm{~mm} /$ sec. After successful closed or open mitral valvotomy it increases but remains slower than normal. Thickening and rigidity of the mitral valve are often associated with calcification. The interfaces produced reflect the ultrasound beam and many echoes return to the transducer. It is possible to determine by this means which mitral valves are thickened and scarred and require surgical replacement and which valves remain pliable and mobile and so are suitable for closed mitral valvotomy. By contrast the anterior cusp echogram in cases of pure non-rheumatic mitral regurgitation (as occurs when mitral chordae rupture) is completely different. The valve cusps are thin, the amplitude of movement is increased, and the diastolic closure rate is very rapid, sometimes in excess of $300 \mathrm{~mm} / \mathrm{sec}$.

Dr. R. B. Pridie described mitral echograms in conditions other than mitral valve disease. In aortic regurgitation rapid oscillation of the anterior mitral cusp is frequently visible on the U.C.G. ${ }^{5}$ Many of these patients have mid-diastolic mur- 
murs not due to mitral stenosis but simulating it (the Austin Flint murmur), and the U.C.G. permits differentiation. In case of severe aortic regurgitation of acute onset the rapid overfilling of the left ventricle due to aortic reflux can produce an early rise in left ventricular diastolic pressure, which causes the mitral valve to close in diastole well before the left ventricle starts to contract. This premature closure of the mitral valve curtails diastolic inflow from the left atrium, reduces cardiac output, and indicates an urgent need for aortic valve replacement. ${ }^{513}$

The U.C.G. provides an accurate but not particularly sensitive method of detecting pericardial effusion. Dr. Ramsey Benham described how fluid may be sought either anteriorly or posteriorly to the heart. Its detection depends on finding an echo-free space external to the free wall of the heart. Dr. Benham recommends looking in front of the heart for fluid. When a pericardial effusion is present the immobile anterior chest wall is separated from the moving surface of the right ventricle by a compressible, fluid-filled space. This echo-free gap varies in width with the cardiac cycle. ${ }^{14}$

Study of the cavity and walls of the left ventricle was described by Dr. D. G. Gibson. He found that the distance between the endocardium of the ventricular septum and the endocardial surface of the posterior wall of the left ventricle measured by U.C.G. bears a predictable but empirical relationship to the volume of the left ventricle measured angiographically. The cube of the U.C.G. dimension approximates to the angiographic volume both at the end of systole and at the end of diastole. ${ }^{9}$ U.C.G. can also be used in the study of localized abnormalities of wall movement caused by conduction defects or local disease. ${ }^{10}$

The modern uses of U.C.G. have been extolled in these columns before. ${ }^{15}$ As its technology improves its applications multiply at an astonishing rate, and it can be compared with electrocardiography 30 years ago. In only a few years it has become indispensable to many cardiologists in permitting the clinic investigation of patients who would have otherwise required admission for more arduous or relatively hazardous invasive investigations.

It is of special value in hospitals with a cardiologist but without facilities for cardiac surgery as a screening method for patients who are being considered for further examination in a specialist unit or for operative treatment. In this way it should speed diagnosis, save unnecessary referrals to other hospitals, reduce time spent in hospital, and limit the more hazardous, uncomfortable, and expensive invasive diagnostic procedures.

At the symposium it was possible only to deal with the better established uses of U.C.G. and some of the most exciting new developments could not be covered. Congenital heart disease was omitted from discussion, but U.C.G. is now being used in the initial screening of newborn babies as well as in the recognition of many congenital cardiac abnormalities. ${ }^{6-8}$ In the newborn it permits recognition of the two most common conditions which present in the first week of life. They are transposition of the great arteries (these babies require to be taken straight to the catheterization laboratory for therapeutic balloon septostomy) and hypoplastic left heart (uncorrectable and soon fatal). In the diagnosis of single ventricle U.C.G. is probably more accurate than angiocardiography. In the young child the number and disposition of the cardiac chambers and of atrioventricular and semilunar valves are fundamental to the diagnosis and can be accurately perceived by U.C.G.

This meeting was the first teaching symposium to be held by the British Cardiac Society, and Dr. Arthur Hollman is to be congratulated on its organization. Others should surely follow and may be attended, it will be hoped, with the same success.



While polio vaccines have largely eliminated poliomyelitis, influenza vaccines have certainly not eliminated influenza, and some people think they are of little use. This view can arise from a misunderstanding in that cases of clinical influenza which occur outside influenza epidemics are not usually due to the influenza A or B viruses contained in the vaccine, and therefore cannot be prevented by its use. Nevertheless, the deaths and severe illnesses which occur during epidemics run into thousands and it should be possible to reduce these by the judicious use of vaccine.

In some ways influenza vaccines have improved considerably in recent years. At one time production was delayed by the time taken to adapt new strains to growth in the chick embryo. Now hybrids between the new strains of influenza $A$ and laboratory strains are prepared, and these grow to high concentration in eggs. ${ }^{1}$ This means that production can be started earlier. The virus contained in the fluids produced is better purified than in the past because of the introduction of zonal centrifugation. ${ }^{2}$ The antigenic composition has to be constantly updated so that the antigens included in each dose correspond with the viruses found in epidemics. The present vaccines contain the $\mathrm{A} / \mathrm{Eng} / 42 / 72$ strain, which has replaced the variant of Hong Kong serotype throughout the world, and also a new B/Hong Kong/5/73 strain, ${ }^{3}$ which might cause the next influenza $B$ epidemic. If given at an appropriate time-a few weeks to a few months before the epidemic-these vaccines would give substantial but not complete protection, provided there is no unexpected antigenic change in the viruses circulating in the community. Since influenza epidemics, if they occur, usually do so between January and April, it would be appropriate to give vaccine about now. It should be given to people at risk of taking the disease badly, particularly the elderly, and those with chronic chest or other diseases such as diabetes. The virus strains causing recent cases of influenza $\mathrm{A}$ in Britain and those which caused outbreaks in Australasia are antigenically different from the A/Eng/42/72 type. However, the present vaccine is likely to give protection against this virus.

The present vaccines still have disadvantages and research is trying to overcome them. For example, intact virus particles have toxic effects, so vaccines made of virus split by detergents are available. The advantage conferred is not very noticeable. They still contain components of the host and the internal 